\title{
Physiological Benefits of Exercise Programs for Youths with Down Syndrome. A Systematic Review
}

\author{
Camacho $\mathrm{R}^{1 * *}$, Castejón-Riber $\mathrm{C}^{2}$, Camacho $\mathrm{J}^{1}$, Requena $\mathrm{F}^{1 * *}$, de Miguel-Rubio $\mathrm{A}^{3}$, Rubio $\mathrm{MD}^{1}$, \\ Escribano $\mathrm{BM}^{1}$ and Agüera $\mathrm{EI}^{1 *}$ \\ ${ }^{1}$ Department of Cellular Biology, Physiology and Immunology. University of Cordoba, Spain \\ ${ }^{2}$ Department Artistic and Corporal Education. Universidad de Cordoba, Spain \\ ${ }^{3}$ Department of Nursing, Pharmacology and Physiotherapy, Spain \\ *Corresponding author: Agüera EI, Department of Cellular Biology, Physiology and Immunology. University of Cordoba, Spain \\ **Co-first authorship: Camacho R and Requena F, Department of Cellular Biology, Physiology and Immunology. University of \\ Cordoba, Spain
}

\section{ARTICLE INFO}

Received: 幽 October 21, 2020

Published: 㓞 October 28, 2020

Citation: Camacho R, Castejón C, Camacho J, Requena F, Agüera EI, et al., Physiological Benefits of Exercise Programs for Youths with Down Syndrome. A Systematic Review. Biomed J Sci \& Tech Res 31(3)2020. BJSTR. MS.ID.005105.

Keywords: Intellectual Disability; Physical Fitness; Biomarkers; Oxygen Consumption; Antioxidant Effect; Lipid Peroxidation

\begin{abstract}
Abstract: Exercise and physical activity provide physiological benefits as well as improvement of physical limitations (i.e. motor ability, strength, agility) in people with Down Syndrome (DS). The aim was to verify if different exercise programs improve physiological parameters response in this population using health markers associated with daily physical activity and physical exercise. The search was carried out in SPORTDiscus, Scopus, PubMed, Medline, the Physiotherapy Evidence (PEDro), and PsychINFO databases. The methodological quality and risk bias of the studies was assessed using the PEDro scale. Eight studies were included in this systematic review. Aerobic training increases maximum oxygen consumption (VO2max) and high-density lipoprotein HDL cholesterol while significantly reducing oxidized low-density lipoprotein (ox-LDL) cholesterol, malondialdehyde, allantoin and allantoin/uric acid ratio. To increase cardiorespiratory fitness, decrease oxidative stress levels and improve lipid profiles and antioxidant capacity, it would be advisable to use an aerobic training program for a minimum of 12 weeks duration, with three sessions/week at $60-75 \%$ of heart rate maximum (HRmax), 30 -minutes session. In case of need, short rests must be included. Researchers should focus on an in-depth study of lipid profiles in this population, because although beneficial results have been observed, it is true that the effects on lipid metabolism are reversible and may disappear.
\end{abstract}

\section{Introduction}

Down Syndrome (DS) is a chromosomal irregularity assignable to total or partial trisomy of Chromosome 21 (C21) which carries enormous medical and social costs [1] (Kamezie al., 2016). It is classified as the most prevalent genetic abnormality in the world $[2,3]$. The severity and range of the syndrome vary significantly as result of an adaptative cellular response to stress [4]. Physical exercise affects people with DS just as it does the general population; it has a direct effect on the great number of physiological problems that influence their health [5]. This study focuses on physiology, given that physiological parameters indicate functional modifications caused by exercise or physical training, and because exercise constitutes an excellent model to exhibit different homeostatic mechanisms. Exercise also helps people maintain a healthy weight [6]. This area of study is important because functional capacity and exercise are inversely correlated with cardiovascular failure [7] as well as with long-term mortality caused by cardiovascular and neoplastic disease [8].

People with DS often have a very sedentary lifestyle which can affect their health [9]. This behavior has been linked to heart impairments, overweight, hypotonicity, gross motor impairments and lack of transportation [10]. Furthermore, Banky and Shields (2011) identified

1) Reduced physical or behavioral skills;

2) Characteristics commonly associated with DS

3) A lack of accessible programs and 
4) Competing family responsibilities as impediment to enroll in physical activity in children with DS. At present, the life expectancy of people with DS is higher than at the end of the twentieth century, reaching the age of 63 and older [11]. Improved access to medical care, particularly in dealing with congenital gastrointestinal and cardiac diseases, has influenced this increase in life expectancy [12]. More than $65 \%$ of this population is overweight [13], which has been shown to cause an increase in mortality due to diabetes [14]. Overweight is a barrier for young people with DS to enter and remain in training programs [15]. A change in lifestyle, including physical exercise results in weight loss, avoids cardiovascular risk and prevents type 2 diabetes [16-18].

Exercise and Physical activity are dissimilar concepts. Exercise is structured, planned and repetitive, with the established objective of improving given physical qualities such as strength, power, speed, and aerobic endurance, among others. On the other hand, physical activity mentions to all movements of the body that consume energy, i.e. daily activities such as walking, gardening, climbing stairs, etc. Nevertheless, physical activity can range from light to vigorous intensity, including activities like swimming or running [19]. Moreover, exercise and physical activity also contribute to an increase in self-determination and motivation for people with this syndrome, providing physiological and psychosocial benefits as well as improvement in physical limitations (i.e. motor ability, strength, agility) [20,21].

In DS, the imbalance of gene expression cause by extra chromosome leads to a series of identifying indicators such as facial features, brachycephaly, clinodactyly, heart, lung, and digestive pathologies, and intellectual disabilities, among others [22]. Some of these features can affect the practice of physical exercise, since they cause reduced exercise capacity in this population; for instance, deficient cardiorespiratory condition compared to persons of the same age and sex without DS [23,24], hypertension, hypercholesterolemia, obesity, etc. [25]. The evaluation of these physiological parameters is an objective way of discovering the body response to different exercise programs. They are also health markers associated with daily physical activity and physical exercise [26]. The cardiorespiratory system is responsible for capturing, transporting and using oxygen during physical activity.

Maximum oxygen consumption (VO2max) is described as the maximum combined capability of the cardiovascular, pulmonary, and muscular systems to intake, carriage and use oxygen, respectively [27]. In aerobic exercise (treadmill, elliptical training, walking, cycling, rowing, swimming, stair climbing), the mode, intensity, duration and frequency of the activity must all be taken into account. Studies have shown that in the prevention of mortality and cardiovascular disease, intensity is more effective than duration $[28,29]$. In 2015, Wee et al. studied the effect of DS and obesity on aerobic capacity and peak heart in adults and youths. Their results suggested that DS decreases equally heart rate peak (HRpeak) and $\mathrm{VO}_{2}$ peak, in spite of age group and obesity severity. Nevertheless, obesity was related to minor $\mathrm{VO}_{2}$ peak in all adults, although not in children with DS.

The lipid profile comprises the analysis and quantification of a series of lipids transported in the blood by different types of plasma lipoproteins. The determination of these parameters is an analytical procedure which is basic to diagnosis and treatment of primary or secondary metabolic disorders. Among the analytical parameters which can be so determined are total cholesterol, cholesterol transported by Low-Density Lipoproteins (LDL), cholesterol transported by High-Density Lipoproteins (HDL), total triglycerides, certain apoproteins, etc. Elevated cholesterol levels are connected with risk of cardiovascular diseases, especially those linked to LDL. Since HDL is that fraction of cholesterol which is transported to the liver for metabolization and excretion by the biliary system, it is not associated with risk of disease [30]. People with DS have a higher risk of dyslipidemia, with a consequently greater risk of cardiopathy. While some authors have stated a low prevalence of atherosclerotic abnormalities in adults with DS, which could decrease the risk of coronary events [31], other researches reveal that these adult patients have a four times greater risk of mortality by ischemia, cardiac disease and cerebrovascular events than the people without DS [32]. In addition, [33] assessed the frequency of dyslipidemia in adolescents and children with DS and found a high incidence of this pathology, nearly 60\%. Therefore, lipid profiles must be performed in patients with DS from a young age, regardless of the risk factors for dyslipidemia. In order to control and reduce cholesterol levels, the custom of physical exercise could be a favorable strategy [34].

The formation of free radicals is a normal and irrevocable process in the body due to chemical reactions in the cell. These radicals introduce oxygen into the cells, producing oxidation of their components and alterations in DNA [35]. It is commonly believed that the Superoxide Dismutase (SOD) (an antioxidant enzyme) catalyzes the dismutation of superoxide anion (02-) in hydrogen peroxide $\left(\mathrm{H}_{2} \mathrm{O}_{2}\right)$, which is subsequently transformed into water through enzymatic mechanism of enzymes like Glutathione Peroxidase (GPX) and catalase. Oxidative stress in people with DS has been linked to trisomy of the $21^{\text {st }}$ chromosome, occasioning the DS phenotype in addition to immune disorders, intellectual disability, biochemical abnormalities, various morphological abnormalities and premature aging [36].

The cause of the upsurge in Reactive Oxygen Species (ROS) may be due to over-expression of the SOD gene, as well as an imbalance between SOD activity and GPX and CAT activity [37]. This lack of balance between the antioxidant and pro-oxidant states would produce an increase in stress in DS; this may affect the mitochondria which are the main origin and target of ROS [38,39]. Moreover, mitochondria are the organelles in which Adenosine Triphosphate 
(ATP) is generated, and the chronic and continued production of ROS causes a decrease in the synthesis of ATP, which occurs during cellular aging. This review adds a combination of 'physiological parameters' such as some blood analyses assessing objectively and quantitatively the consequences of the training program on participants. The study of these physiological systems simplifies the evaluation of health markers associated with daily physical activity and physical exercise (Ortega et al., 2019). Consequently, the purpose of the current review is to verify if different exercise programs improve cardio-respiratory, lipid and antioxidant responses in young individuals with DS using health markers associated with daily physical activity and physical exercise

\section{Materials and Methods}

\section{Search Strategy}

A literature search was carried out in SPORTDiscus, Scopus, PubMed, Medline, the Physiotherapy Evidence (PEDro), and PsychINFO databases. Our search was restricted to the years between 2000 and 2018. In bibliometric terms, it is known that for a search to be considered properly carried out, it must be based on articles published in the last five years [40]. However, since in the search we found articles published between 2000-2013 that demonstrate the importance of a training program on physiological parameters (heart rate and catalase, malondialdehyde, allantoin and uric acid activity) in the population with DS, we decided that it was important to include them in this review and thus searched back to the year 2000. To begin with, we created four categories of keywords related to

i. DS

ii. Sport

iii. Physiological parameters related to exercise and

iv. Intervention. The next descriptors terms combined with Boolean operators were used: ("Down syndrome" OR "trisomy 21" OR "chromosome 21" OR "intellectual disability") AND ("sport" "OR "physical fitness" OR "training program") AND ("physiological parameters" OR "peroxidation" OR "oxygen consumption" OR "antioxidant capacity") AND ("intervention"). The indicators of the PRISMA statement were followed.

\section{Selection Criteria}

The inclusion criteria for article selection were studies published between 2000 and 2018 in Southern Europe; participants under 25 years of age diagnosed with DS; intervention via a physical training program, and objectives related to physical capacity and physiological assessment. The exclusion criteria were articles published before 2000; DS adults over 25 years old; participants with intellectual disabilities in whom other diagnoses were mixed with DS and results were not presented separately; objectives not related to exercise and assessment of physiological parameters.

\section{Study Selection Process}

Article screening was conducted independently by three reviewers (R.C., J.C., and F.R.). Once the systematic search was achieved, the screening method consisted on exclude duplicates articles, then review titles/abstracts and excluding those articles did not gathered selection criteria. If it was not clear whether the article should be incorporated, the entire paper was acquired to verify that it met all the inclusion criteria. If the three authors did not agree, another reviewer (E.I.A.) helped them reach an understanding. At the next stage, the outstanding article were evaluated in detail screening full-text articles using criteria for inclusion. Three authors extracted the relevant information from the chosen articles in a systematic and gradual way to ensure accuracy. The first (R.C), third (J.C.) and fourth (F.R.) author made a summary chart that classified the results, considering the main physiological response in each study. The summary was emailed to all authors in order to discuss the analysis of search results and the data extraction chart in a later video conference (Zoom Platform). A total of 36 articles met the selection criteria, of which 28 were excluded with justification and 8 selected by unanimous agreement.

\section{Data Extraction}

The first (R.C), third (J.C) and fourth (F.R.) author extracted the following data from each article chosen for this review and reaching at an agreement on all items. The next data was obtained from the articles:

1) Year of publication,

2) Author,

3) Sample size,

4) Participant demographic characteristics (region, gender, age, height, weight),

5) Intervention type,

6) Duration and frequency,

7) (material used),

8) (physiological parameter evaluated)

9) Pre and post results and

10) Physiological response to exercise in each study.

\section{Assessment of Risk of Bias}

So as to assess the methodological quality of studies, the PEDro scale (Maher et al., 2010) was used. This scale consists of a checklist of 11 scored yes ( 1 point)-no ( 0 point) questions related to selection, performance, detection, information and attribution base. Score 1 is not used for the calculation of total score of the scale. The higher the score obtained, the greater the methodological quality of the assessed study (range score=0-10). A study with a score from 6 
to 10 is considered as evidence level 1(6-8: good; 9-10: excellent) and a study with a score from 5 to 0 is considered as evidence level 2 (4-5: acceptable; < 4: poor) [41]. This process implicated a separate analysis by two evaluators (R.C. and F.R.). When there was no consensus, J.C. reviewed the study until the classification was confirmed. The final ranking of every study in each scale was reached by unanimity in a video conference discussion.

\section{Type of Intervention}

The intervention programs assessed were: rowing ergometer [42]; jogging and walking [43]; an increasing 12-week training program [44,45] swimming, athletics, volleyball, handball, basketball and football [25]; a strength-training circuit for the lower and upper body: bench press, front pulley, seated row, leg press, leg and ankle curls [46]; jumps, wall push-ups, fitness bands and medicine balls [24] (Table 3).

\section{Obsolescence Assessment}

Two bibliometric indicators, Burton and Kebler's "half-life" and
Prince Index, was evaluated by the fist (R.C.) and fourth F.R.). Burton and Kebler's "half-life" (age or obsolescence) is the difference between the publication date of the article or publication analyzed; and the Price Index, that is, the percentage of references that are less than five years old (ratio between the number of references with less than 5 years and the total number of references for that year, multiplied by 100 (PI=(references-5 years)/total X100) [47]. The descriptive analysis was performed using an Excel spreadsheet.

\section{Results}

\section{Study Characteristics}

Figure 1 shows each step of the screening method. A total of 441 articles were found from the preliminary exploration as possibly significant articles. No extra articles were recognized through other sources. Once duplicates were removed, 397 were screened by title and abstract and 361 excluded. The authors did a full-text screening of 35 articles; 27 were eliminated with justification, resulting in 8 studies aimed at determining the influence of training on cardiorespiratory, lipid and antioxidant response in young individuals with DS (Figure 1).

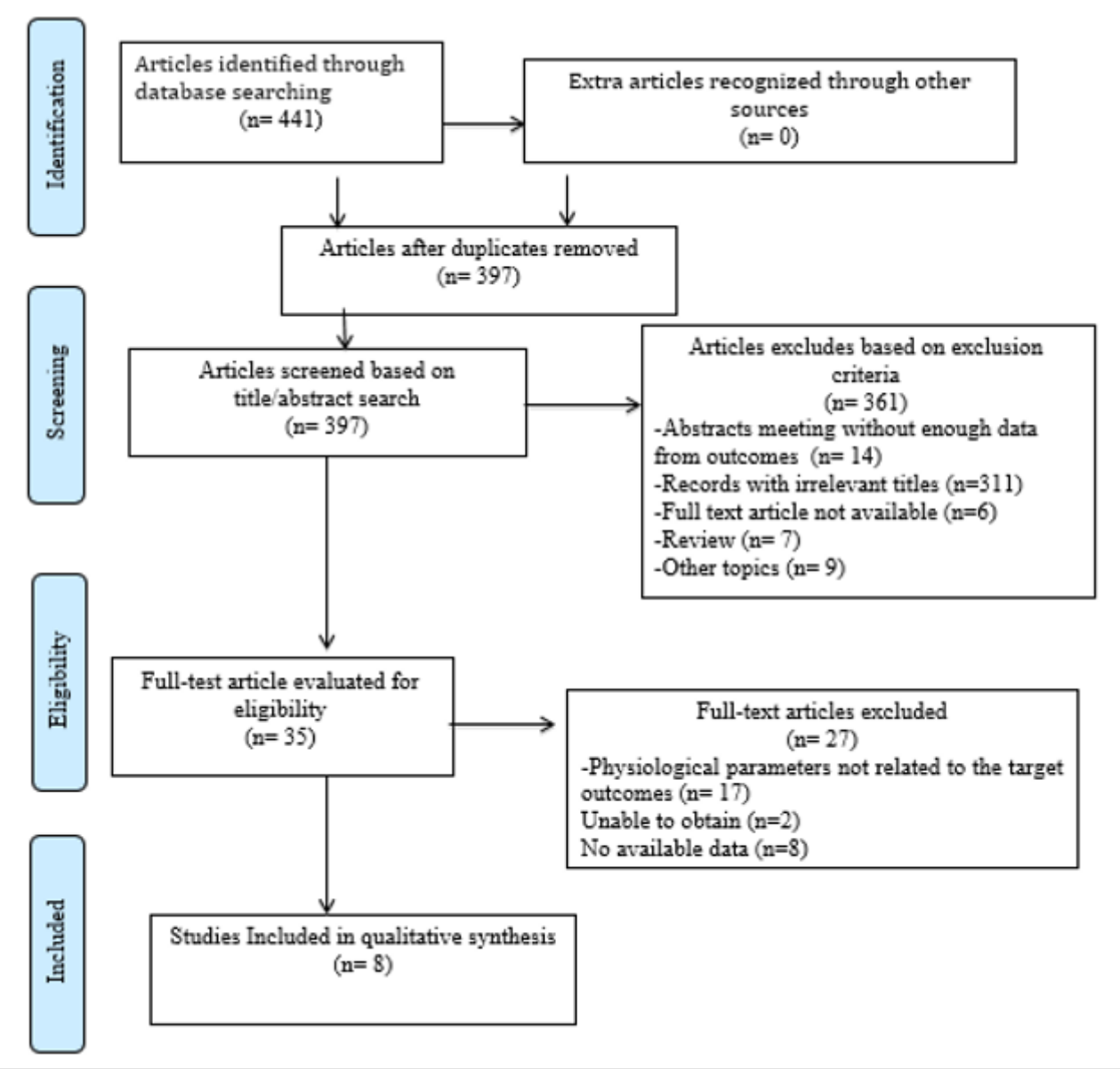

Figure 1: Flowchart of screening methods. 


\section{Risk Bias}

The scores of the Physiotherapy Evidence Database scale for each study incorporated in this review is shown in Table 1. Five of the studies $[43-46,48]$ achieved a score of 6 , therefore they were considered to have high methodological quality [41]. None of the studies made use of a system of concealment of group assignment. Due to 7 studies [24,42-46,48] evaluated physical training programs in a control group and in an intervention group, the therapist and participants could not be blinded in any study. The lowest score gotten was $5[24,42]$. One study was not evaluated [25]. With respect to possible bias in the articles included in this review, no study assigned blinded treatment. Of all types of bias detected, the greatest were performance bias and detection bias; none of the studies blinded participants. Attrition bias was detected in two articles [24,42]. As for the remaining criteria described on the PEDro scale, results were satisfactory (PEDro).

Table 1: Physiotherapy Evidence Database (PEDRo) scale. Scores acquired after methodological evaluation of studies included in the review.

\begin{tabular}{|c|c|c|c|c|c|c|c|c|c|c|c|c|c|}
\hline \multirow[b]{2}{*}{ Study } & \multicolumn{13}{|c|}{ PEDRo Scale } \\
\hline & 1 & 2 & 3 & 4 & 5 & 6 & 7 & 8 & 9 & 10 & 11 & Total & $\begin{array}{l}\text { Methodological } \\
\text { quality }\end{array}$ \\
\hline Varela et al., (2001) & - & Yes & No & Yes & No & No & No & Yes & No & No & Yes & 5 & Acceptable \\
\hline $\begin{array}{l}\text { Tmisaras et al., } \\
\text { (2003) }\end{array}$ & - & Yes & No & Yes & No & No & No & Yes & Yes & Yes & Yes & 6 & Good \\
\hline $\begin{array}{l}\text { Rosety-Rodríguez el } \\
\text { al., (2006) }\end{array}$ & - & Yes & No & Yes & No & No & No & Yes & Yes & Yes & Yes & 6 & Good \\
\hline $\begin{array}{l}\text { Ordoñez \& Rosety- } \\
\text { Rodríguez (2007) }\end{array}$ & - & Yes & No & Yes & No & No & No & Yes & Yes & Yes & Yes & 6 & Good \\
\hline $\begin{array}{l}\text { Rosety Rodriguez et } \\
\text { al., } 2010\end{array}$ & - & Yes & No & Yes & No & No & No & Yes & Yes & Yes & Yes & 6 & Good \\
\hline Casajus et al., 2012 & - & - & - & - & - & - & - & - & - & - & - & - & - \\
\hline $\begin{array}{l}\text { Rosety Rodríguez et } \\
\text { al., } 2013 \\
\end{array}$ & - & Yes & No & Yes & No & No & No & Yes & Yes & Yes & Yes & 6 & Good \\
\hline $\begin{array}{l}\text { Gonzalez-Agüero et } \\
\text { al., } 2014\end{array}$ & - & Yes & No & Yes & No & No & No & Yes & No & Yes & Yes & 5 & Acceptable \\
\hline
\end{tabular}

Note: *The PEDro scale was not used to assess the quality of Casajus et al., (2012) because this scale only allows for randomized Controlled Tests. Therefore, this study was not evaluated.

\section{Data Extraction}

Eight studies with a total of 241 participants (intervention group (IG) $n=173$; control group (CG) $n=68$ ) were included in this review. Respecting the number of participants, the smallest sample size was reached by [24] $(n=14)$, in while the highest number of participants was observed by [46] $(n=40)$. With respect to geographic region

Table 2: Demographic details of participants. where the study was conducted, 6 out of 8 were carried out in Spain $[24,25,44-46,48]$ one in Portugal [42] and the other in Greece [43]. As regard gender, only two studies [24,25] included men and women in their intervention group. Regarding participant age, the highest mean values (24.6 years) were recorded in the study of [43], while the lowest (14) were found in the study carried out by [25]. Demographic characteristics are shown in Table 2.

\begin{tabular}{|c|c|c|c|c|c|c|}
\hline Studies & Participants (n) & Region & Gender & Mean age & Mean height & Mean Weight \\
\hline Varela et al., (2001) & $\begin{array}{c}\text { N=16; } \\
{ }^{*} \mathrm{CG}: 8 \\
\text { IG:8 }\end{array}$ & Portugal & Male & $21.4 \mathrm{yr}$. & $156.3 \mathrm{~cm}$ & $62.6 \mathrm{Kg}$ \\
\hline $\begin{array}{l}\text { Tmisaras et al., } \\
\text { (2003) }\end{array}$ & $\begin{array}{c}\mathrm{N}=25 ;{ }^{*} \mathrm{CG}: 10 \\
\mathrm{IG}: 15\end{array}$ & Greece & Male & & & $72.3 \mathrm{Kg}$ \\
\hline $\begin{array}{l}\text { Rosety-Rodríguez } \\
\text { el al., (2006) }\end{array}$ & $\begin{array}{l}\text { N=38; } \\
\text { *CG:7 } \\
\text { IG:31 }\end{array}$ & Spain & Male & $16.3 \mathrm{yr}$. & No data & No data \\
\hline $\begin{array}{l}\text { Ordoñez \& Rosety- } \\
\text { Rodríguez (2007) }\end{array}$ & $\begin{array}{l}\text { N=16; } \\
{ }^{*} \mathrm{CG}: 7 \\
\mathrm{IG}: 31\end{array}$ & Spain & Male & 16.3 & $155.2 \mathrm{~cm}$ & $70.8 \mathrm{Kg}$ \\
\hline
\end{tabular}




\begin{tabular}{|c|c|c|c|c|c|c|}
\hline $\begin{array}{l}\text { Rosety Rodriguez } \\
\text { et al., } 2010\end{array}$ & $\begin{array}{l}\text { N=38; } \\
* \text { CG:7 } \\
\text { IG:31 }\end{array}$ & Spain & Male & $16.3 \mathrm{yr}$ & $155.2 \mathrm{~cm}$ & $70.8 \mathrm{Kg}$ \\
\hline Casajus et al., 2012 & $\begin{array}{c}\mathrm{N}=19 \\
\text { CG:0 } \\
\text { IG:19 }\end{array}$ & Spain & $\begin{array}{c}10 \text { females } \\
9 \text { males }\end{array}$ & 14 yr. & $133.7 \mathrm{~cm}$ & $38.8 \mathrm{Kg}$ \\
\hline $\begin{array}{l}\text { Rosety Rodríguez } \\
\text { et al., } 2013\end{array}$ & $\begin{array}{c}\mathrm{N}=40 \\
{ }^{*} \mathrm{CG}: 16 \\
\mathrm{IG}: 24\end{array}$ & Spain & Male & $23.7 \mathrm{yr}$. & No data & $63 \mathrm{~kg}$ \\
\hline $\begin{array}{l}\text { Gonzalez-Agüero et } \\
\text { al., } 2014\end{array}$ & $\begin{array}{c}\mathrm{N}=27 \\
{ }^{*} \mathrm{CG}: 13 \\
\text { IG:14 }\end{array}$ & Spain & $\begin{array}{l}12 \text { females } \\
15 \text { males }\end{array}$ & $14.5 \mathrm{yr}$. & No data & No data \\
\hline
\end{tabular}

Note: CG: control group; IG: intervention group *CG: continued with normal activities; They did not partake in any routine physical training. yr: year.

All studies [42-46,48,24,25] investigated the physiological response of exercise programs. Five out of eight studies described a 12-week interval treadmill training programmed [43-46, 48,25] one study increasing speed (1 km.hr-1) and time every minute until volitional exhaustion [43]; three studies increasing intensity and time every three weeks $[44,48,45]$. One study described 16week rowing ergometry training regimen, increasing slope every two minutes and sped every 1 minute [42]. One study used a 30week program based on sport-games (athletics, handball, football, basketball or volleyball) and swimming [25]. Two studies used circuit training, one including a 21-week plyometric jumps circuit increasing set of series and set of repetitions [24] and other including 12 week-six station lower and upper body activities circuit [46].

Regarding the frequency of these interventions, six studies reported 3 times per week [42-46, 48] however, two studies reported twice per week [25,24]. With respect to control group, seven studies $[42-46,48,24]$ had a control group and one study had none [25]. All studies [42-46,48,25,24] divided the intervention into phases: warm-up, exercise and cool-down.

Concerning the expected physiological response, four studies $[42,43,24,25]$ evaluated cardio-respiratory capacity. One study [42] reported that cardiovascular fitness (V02peaks) did not improve, but the participants gained in work performance. However, three out of four studies reported a positive outcome for VO2max [24, $25,43]$. Three studies assessed the efficiency of training programs on catalase, malondialdehyde and allantoin in other ways in order to evaluate the improvement of antioxidant systems $[44,45,48]$.

In contrast with the baseline, the values of the catalase activity after a 12- week training program did not increase significantly-1,607.0 (231) U/g Hb vs 1,663.2 (280) U/g Hb; $(p=0.151)-$. No significant differences were found in controls. On the other hand, on study [48] fond that although the amount of malondialdehyde diminished significantly $(0.41 \pm 0.1 \mathrm{e} 2$ vs. $0.32 \pm 0.09 \mu \mathrm{mol} \cdot \mathrm{L}-1 ; \mathrm{p}=0.011$ ) in exercise. In contrast, controls had no significant differences in malondialdehyde levels once compared to baseline $(0.42 \pm 0.10$ vs. $0.43 \pm 0.09 \mu \mathrm{mol} \cdot \mathrm{L}-1 ; \mathrm{p}=0.64)$. One study [45] reported that plasmatic levels of allantoin were reduced significantly (22.09 (1.62) vs 18.74 (1.38) mmol/l; p,0.001) afterward being exercised. Furthermore, it was described that the allantoin/uric acid ratio was reduced significantly $(0.071$ (0.006) vs 0.059 (0.004); p,0.05). No change was stated in control. One study [46] lipid peroxidation. Plasma levels of oxLDL were significantly diminished following the end of the training program $(5228,2 \pm 2144,7$ vs $4760,1 \pm 2024,6 \mathrm{ng} / \mathrm{ml} ; \mathrm{p}=0,0118 ; \mathrm{d}=0,96)$. Serum lipid profile and fat mass were significantly improved too $(30,1 \pm 2,9$ vs $27,8 \pm 2,3 \% ; p=0,038 ; d=0,82)$. Four studies [42, 43, $46,24]$ reported familiarization period before test. Three studies determined oxygen uptake and respiratory exchange ratio handling an open-circuit spirometry system [25,42,43]. Four studies reported telemetry data recorder [25,42,43] Gonzalez-Agüero, et ., 2014 and four studies [44-46,48] predicted the maximal heart rate by the equation HRmax=194.52(0.56 age).

Five studies [43-46,48] targeted HR for a moderate intensity between $60-75 \%$ peak heart rate or peak oxygen uptake; one study [42] targeted HR between (55-70\%); however, it was not described in the other two studies [25]; González-Agüero 2014). All articles from the years 2000 to 2018 were reviewed, with an obsolescence (measured by the mean) of 8 years (Burton Kebler index) and a Price index (percentage of articles less than 5 years old) of 25\%. Further details of intervention characteristics, parameters investigated, pre/post results and physiological response to exercise of the articles included are shown in Table 3. 
Table 3: Details of characteristics and results of the selected studies.

\begin{tabular}{|c|c|c|c|c|c|c|c|}
\hline AUTHORS & Intervention & Intensity & $\begin{array}{c}\text { Duration/Fre- } \\
\text { cuency }\end{array}$ & Material used & $\begin{array}{c}\text { Physiological } \\
\text { Parameters }\end{array}$ & $\begin{array}{l}\text { Pre/Post re- } \\
\text { sults }\end{array}$ & $\begin{array}{c}\text { Physiological re- } \\
\text { sponse }\end{array}$ \\
\hline $\begin{array}{l}\text { Varela et al., } \\
\text { (2001) }\end{array}$ & $\begin{array}{c}\text { Exercise: rowing } \\
\text { ergometer } \\
\text { - Warmup: } 10 \\
\text { min } \\
\text { - Exercise: } \\
\text { (i) Phase 1: } \\
15 \text { min for } \\
\text { weeks } 1 \\
\text { through } 6 \\
\text { (ii) Phase } 2: \\
\text { Increase } 5 \text { min } \\
\text { each } 2 \text { weeks up } \\
\text { to } 25 \text { min } \\
\text { (iii) Phase } 3 \text { : } \\
\text { Keep on at } 25 \\
\text { minutes. }\end{array}$ & $\begin{array}{c}55-70 \% \\
\text { peak } \mathrm{VO}_{2} \\
\text { - From 1st } \\
\text { to } 4 \text { th week: } \\
55-60 \% \\
\text { intensity } \\
\text { - From 5th } \\
\text { to } 16 \text { th } \\
\text { week: } 70 \% \\
\text { intensity }\end{array}$ & $\begin{array}{c}\text { Duration: } \\
16 \text { weeks } \\
\text { Frequency: } 3 \text { ses- } \\
\text { sions/week }\end{array}$ & $\begin{array}{c}\text { Telemetry } \\
\text { Ergopirometry }\end{array}$ & 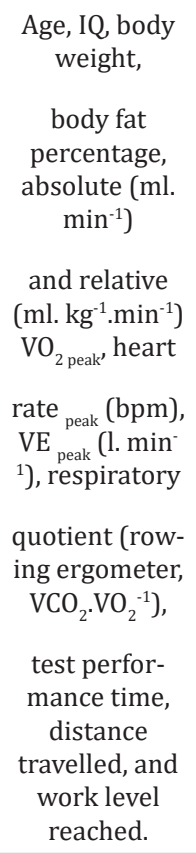 & $\begin{array}{l}\text { Following } \\
\text { training, no } \\
\text { changes in } \\
\text { cardiovascular } \\
\text { fitness had } \\
\text { occurred for } \\
\text { intervention } \\
\text { group. } \\
\text { However, they } \\
\text { reached signifi- } \\
\text { cantly higher } \\
\text { levels of work } \\
\text { performance for } \\
\text { both treadmill } \\
\text { and rowing } \\
\text { ergometer } \\
\text { post-training } \\
\text { tests. p, .01 }\end{array}$ & $\begin{array}{l}\text { The training program } \\
\text { chosen did not im- } \\
\text { prove cardiovascular } \\
\text { fitness (peak } \mathrm{VO}_{2} \text { pe- } \\
\text { aks), but ameliorated } \\
\text { body weight and body } \\
\text { fat percentage. } \\
\text { Time, distance, and } \\
\text { peak resistance } \\
\text { demonstrated signifi- } \\
\text { cant improvements } \\
\text { in the exercise reg- } \\
\text { imen }\end{array}$ \\
\hline $\begin{array}{l}\text { Tsimaras et } \\
\text { al. (2003) }\end{array}$ & $\begin{array}{l}\text { - Warm-up: } 15 \\
\text { min } \\
\text { - Jogging and } \\
\text { walking: } 30 \text { min } \\
\text { (5 jogging } \\
\text { periods of } 4 \text { min } \\
\text { each; walking } \\
2 \text { min was } \\
\text { allowed) } \\
\text { - Cool down: } 5 \\
\text { min. }\end{array}$ & $\begin{array}{c}\text { Intensity } \\
\text { 60-75\% HR } \\
\text { max. }\end{array}$ & $\begin{array}{c}\text { Duration: } \\
12 \text { weeks } \\
\text { Frequency: } \\
3 \text { sessions/week }\end{array}$ & $\begin{array}{c}\text { Telemetry } \\
\text { Ergopirometry }\end{array}$ & $\begin{array}{l}\text { Oxygen uptake } \\
\text { Peak Heart } \\
\text { Rate, Peak } \\
\text { minute ven- } \\
\text { tilation, peak } \\
\text { respiratory } \\
\text { exchange } \\
\text { ratio, peak } \\
\mathrm{V}_{02} \text { (absolute } \\
\text { and relative), } \\
\text { and test time } \\
\text { during tread- } \\
\text { mill test. }\end{array}$ & $\begin{array}{l}\text { After exercise: } \\
\text { IG significantly } \\
\text { > values than } \\
\text { GC on peak } \\
\text { VE, V02peak } \\
\text { (absolute and } \\
\text { relative), and } \\
\text { test time } \\
\text { (p <.05). } \\
\text { Post-training: } \\
\text { IG enhanced } \\
\text { peak VE (p< } \\
.05 \text { ), V02 peak } \\
\text { (p <.05 and p < } \\
.01 \text { in absolute } \\
\text { and relative, } \\
\text { respectively), } \\
\text { and test time (p< } \\
.01 \text { ) in contrast } \\
\text { with their base- } \\
\text { line results. }\end{array}$ & $\begin{array}{l}\text { The training program } \\
\text { chosen improved } \\
\text { cardiorespiratory re- } \\
\text { sponse by increasing } \\
\mathrm{VO}_{2} \mathrm{max} \text {, an important } \\
\text { component for physi- } \\
\text { cal fitness from an aer- } \\
\text { obic point of view. }\end{array}$ \\
\hline $\begin{array}{l}\text { Rosety-Ro- } \\
\text { dríguez et } \\
\text { al., (2006) }\end{array}$ & $\begin{array}{l}\text {-Warmup: } 10 \\
\text { min } \\
\text {-Main activity: } \\
\text { 25-35 min. In- } \\
\text { creasing } 5 \text { min } \\
\text { each } 3 \text { weeks } \\
\text {-Cool down: } 10 \\
\text { min } \\
\text { - Intensity 60- } \\
\text { 75\% theoretical } \\
\text { HR max. }\end{array}$ & $\begin{array}{c}\text { Intensity } \\
60-75 \% \\
\text { theoretical } \\
\text { HR max } \\
\\
\text { HR- } \\
\text { max=194.5- } \\
\text { [0.56 age } .\end{array}$ & $\begin{array}{c}\text { Duration: } \\
12 \text { weeks } \\
\text { Frequency: } \\
3 \text { sessions/weeks }\end{array}$ & $\begin{array}{c}\text { Blood samples } \\
\text { Beutler's method }\end{array}$ & $\begin{array}{l}\text { Catalase } \\
\text { activity. }\end{array}$ & $\begin{array}{c}\text { IG: } 1,607.0 \\
\text { (231) IU/g Hb } \\
\text { vs 1,663.2 (280) } \\
\text { IU/g Hb; } \\
\text { (p = 0.151) } \\
\text { CG: No signifi- } \\
\text { cant differences }\end{array}$ & $\begin{array}{l}\text { The training program } \\
\text { chosen increased } \\
\text { catalase activity, but } \\
\text { not significantly. } \\
\text { Moderate physical } \\
\text { activity decreases oxi- } \\
\text { dative stress. Catalase } \\
\text { creates a fast reaction } \\
\text { against free radicals } \\
\text { of hydrogen peroxide, } \\
\text { converting them into } \\
\text { water and oxygen. }\end{array}$ \\
\hline
\end{tabular}




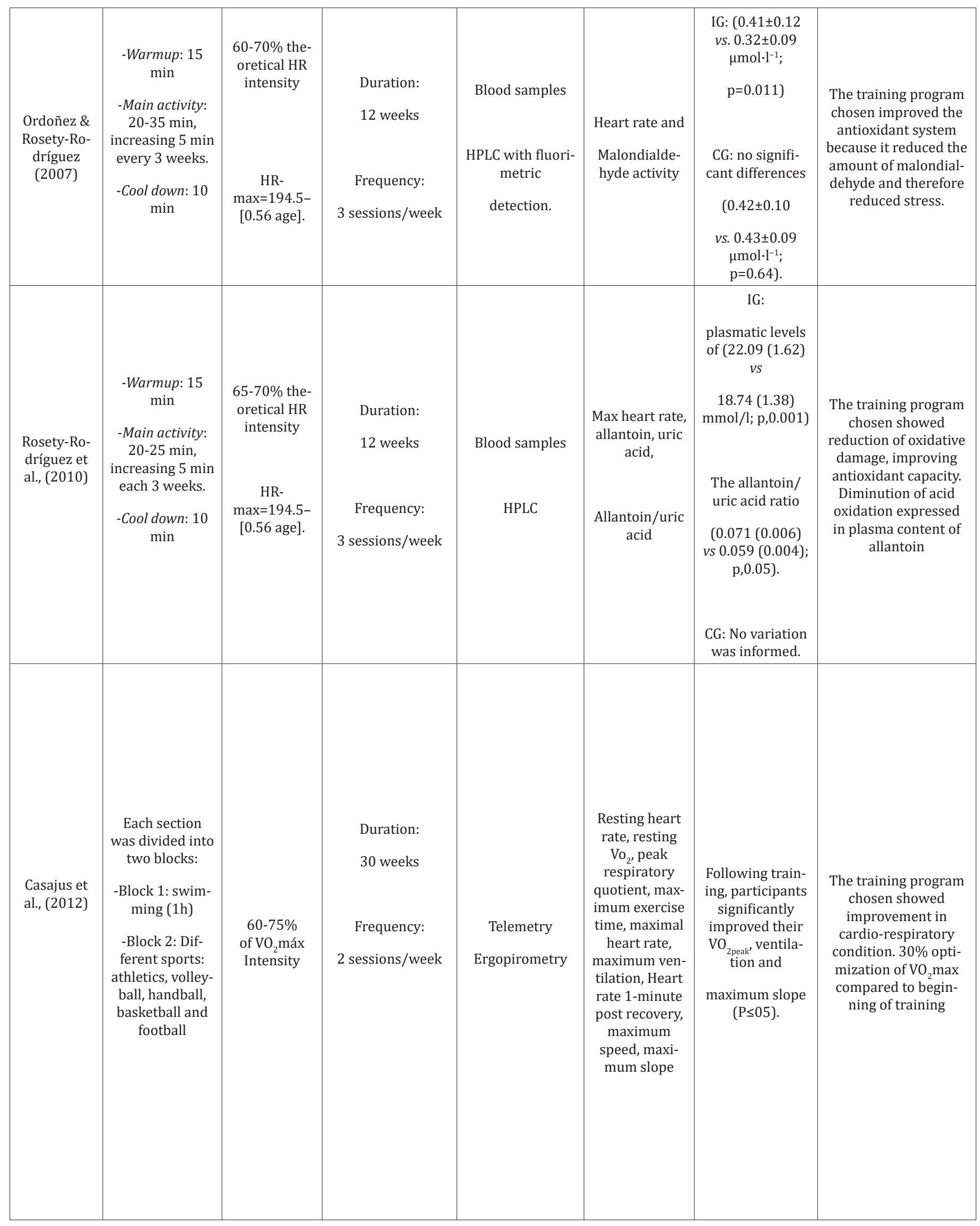




\begin{tabular}{|c|c|c|c|c|c|c|c|}
\hline $\begin{array}{l}\text { Rosety-Ro- } \\
\text { dríguez et } \\
\text { al., (2013) }\end{array}$ & $\begin{array}{c}\text {-Warmup:10-15 } \\
\text { min } \\
\text {-Strength-train- } \\
\text { ing } \\
\text { circuit of } 6 \\
\text { lower and upper } \\
\text { body activities: } \\
\text { bench press, } \\
\text { front pulley, } \\
\text { seated row, leg } \\
\text { press, leg and } \\
\text { ankle curls. } \\
\text { 8 repetitions } \\
\text { each. } \\
\text {-Recovery } \\
\text { period between } \\
\text { session ( } 90 \\
\text { seconds). } \\
\text {-Calm down: } \\
5-10 \text { min }\end{array}$ & $\begin{array}{l}\text { Not de- } \\
\text { scribed }\end{array}$ & $\begin{array}{c}\text { Duration: } \\
12 \text { weeks } \\
\text { Frequency: } \\
3 \text { sessions/week }\end{array}$ & $\begin{array}{l}\text { Blood samples. } \\
\text { Commercial } \\
\text { ELISA-kits }\end{array}$ & $\begin{array}{l}\text { oxLDL } \\
\text { Fat mass and } \\
\text { serum lipid } \\
\text { profile }\end{array}$ & 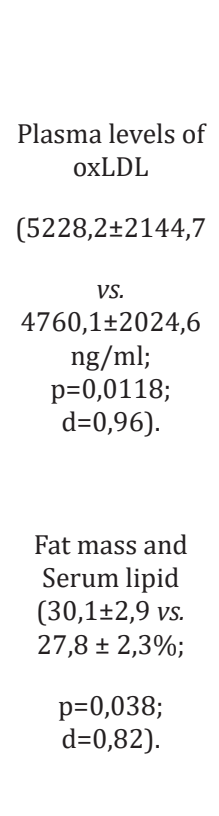 & $\begin{array}{c}\text { The training program } \\
\text { chosen improved lipid } \\
\text { profile and lipoperox- } \\
\text { idation }\end{array}$ \\
\hline $\begin{array}{l}\text { González } \\
\text { Agüero et } \\
\text { al., } \\
\text { (2014) }\end{array}$ & $\begin{array}{l}\text {-Warmup: } 5 \text { min } \\
\text {-Physical } \\
\text { exercise: } 10-15 \\
\text { min (jumps, } \\
\text { wall push-ups, } \\
\text { fitness bands } \\
\text { and medicine } \\
\text { balls). } \\
\text {-Calm Down: } \\
5 \text { min }\end{array}$ & $\begin{array}{l}\text { Not de- } \\
\text { scribed }\end{array}$ & $\begin{array}{l}\text { Duration: } \\
21 \text { weeks } \\
\text { Frequency: } \\
2 \text { days /weeks }\end{array}$ & $\begin{array}{l}\text { Ergospirometry } \\
\text { Electrocardiog- } \\
\text { raphy }\end{array}$ & $\begin{array}{l}\text { Heart rate, } \\
\text { Minute ventila- } \\
\text { tion, respira- } \\
\text { tory exchange } \\
\text { ratio, } \mathrm{V}_{\text {ormax' }} \\
\text { Work time } \\
\\
\text { were mea- } \\
\text { sured pre and } \\
\text { post exercise }\end{array}$ & $\begin{array}{l}\text { CG: improved all } \\
\text { their cardio- } \\
\text { respiratory } \\
\text { parameters } \\
\text { (all p<.05). } \\
\text { IG > values than } \\
\text { CG in all car- } \\
\text { diorespiratory } \\
\text { parameters } \\
\text { after training (all } \\
\text { p<.05). }\end{array}$ & $\begin{array}{c}\text { The training program } \\
\text { chosen } \\
\text { improved cardiore- } \\
\text { spiratory resistance } \\
\text { (VO2max, heart rate, } \\
\text { maximum respiratory } \\
\text { quotient, maximum } \\
\text { ventilation) increased } \\
\text { bone mass and lean } \\
\text { mass }\end{array}$ \\
\hline
\end{tabular}

Note: CG: control group; IG: intervention group; HRmax: Hear rate maximum; $\mathrm{VO}_{2}$ max: Maximal oxygen consumption; Peak VO ${ }_{2}$ peak Maximal oxygen consumption peak; $\mathrm{VCO}_{2}$ : Carbon dioxide production; $\mathrm{VO}_{2}$ : Oxygen consumption; Peak VE: ventilation exchange peak; oxLDL: Oxidized low-density lipoprotein; IU/g Hb: International Units/gram Hemoglobin; HPLC: High performance liquid chromatography; ELISA: Enzyme-Linked ImmunoSorbent Assay.

\section{Discussion}

Different exercise programs enhance the physiological responses of cardio-respiratory, lipid and antioxidant systems in young individuals with DS when performing a well-design and systematic training program. Notwithstanding the extensive literature, only eight articles were selected because they met the inclusion criteria for this review [42-46,48,24,25].

Price's index of fast-growing scientific specialties might be as high as $80 \%[49,50]$. The studies included in this review reached a "half-life" of eight years and a Prince's index of 25\%, which indicates a low scientific growth in the evaluated area. It is very interesting and necessary that researchers continue to delve into the physiological benefits of exercise programs in people with DS. The quality score of five articles incorporated in this review according to PEDro scale was acceptable. With respect to possible bias in the articles included in this review, no study assigned blinded treatment. Of all types of bias detected, the greatest were performance bias and detection bias; none of the studies blinded participants. Attrition bias was detected in two articles [24, 42,]. Only one article [25] was not evaluated by The PEDro scale for not being consider Randomized Controlled Trial. As for the remaining criteria described on the PEDro scale, results were satisfactory (http://www.pedro.org.au).

All studies [24,25,42-46,48] divided the intervention into phases: warm-up, exercise and cool down. We agree that it is essential to include a warm-up phase in this type of intervention, provides neurological, physiological and physiological improvements physiological, preparing the body for the exercise phase [51]. The cool-down phase aids the physiological and psychological normalization of the individual [52]. We suggest the training session should not be finished without a progressive 
cool-down phase in order to lower blood pressure, normalize body temperature, respiration, and heart rate and return to the body's normal metabolic level when not making an extra effort [52]. It is of interest to note that only one of the studies revised [46] reported using stretching exercises in the calm down phase. Other studies $[24,25,42-45,48]$ did not include this data.

Participants in the studies selected ranged in age from 14 to 24 years. First of all, authors decided to include individuals of 25 years and younger based on the WHO's stratification scale of adolescents and youths, which considers people between the ages of 18 and 25 as "young people" and between the ages of 26 and 30 "young adults (WHO). Authors targeted on those ages because adults with DS are less physically active and show greater cognitive deterioration, which has a negative impact on their ability to plan and to comprehend abstract information, delaying decision making [53]. On the other hand, given their experience in the physiology of exercise, some of the authors of this review thought that young adults with DS could improve their cardiovascular condition with well designed, strictly supervised aerobic exercise. The repercussions of improvement in the capturing of oxygen could be fundamental in improving their performance of normal daily activities. Besides, adults with DS are less motivated to participate in this kind of intervention. At the same time, it has been shown that people in this age range have a greater capacity for social interaction during exercise, which encourages them to participate in intervention programs $[54,55]$. Besides, young people in this age range have less difficulty planning and organizing activities and understand the benefits of exercise better (improved health and physical condition, weight loss) [56].

The cardiorespiratory function of people with DS is insufficient; it has been detected that they have a lower VO2max than people without DS [23]. This may be due to

i. maximum HR decreasing cardiac output, and hence $\mathrm{VO}_{2} \max [57,58]$;

ii. Respiratory anomalies suffered by individuals with DS [58]; and

\section{iii. An excessive amount of body fat $[59,60]$.}

Four of the eight revised studies evaluated the aerobic capacity of the participants $[24,25,42,43]$. In the one hand, three of the studies $[24,25,43]$ concluded that the training program chosen ameliorated the cardiorespiratory condition with increasing VO2max. On the other hand, one study [42] described improvements in performance measures without improvement in V02max. In accordance with La Course (2009), the increase in performance should not be underestimated since it will influence an enhance in the capacity to do activities of daily living and therefore in the quality of life. This may be due to the fact that the intensity of the exercise used by $[24,25,43]$ was greater than that used by [42]. Because aerobic training can be done at varying intensities and durations, [42] recognized that physical fitness can not only be enhance with training, but that there are also factors such as motivation for the type of exercise, decrease in anxiety] or acclimation with exercise tests [61]. Also, [24] established not only an improvement in cardio-respiratory endurance parameters in adolescents and children with DS, but also an augmentation in lean and bone mass, which benefits the health of these people. Therefore, we can state that cardiovascular resistance is closely related to an individual's cardiovascular health. The fact that it improves with aerobic training is very important this population has a serious risk of cardiovascular disease as well as diseases related to bone health [62]. [25,29] presenting that young people with DS optimized their V02max by $30 \%$, achieved healthier values resembling those of individuals without DS [63].

However, as they did not contrast their results with a control group, they could not demonstrate that the changes were the effect of training and not a consequence of physiological changes linked to growth. We believe that these results are not of quality because this study had risk of bias. In any case, it has been documented that the VO2 of sedentary children does not improve with growth [64]. The highest percentage of studies carried out a training intervention for 12 weeks, with 3 sessions/week. Due to the physiological limitations of people with DS, such as their low rate of respiratory oxygen exchange, at least 12 weeks of training is needed to improve their aerobic fitness [43-46,48]. Besides, circuit training including plyometrics or resistance improves several cardiorespiratory parameters [24] and lipoperoxidation [46]. Thus, the performance of daily activities would improve, along with autonomy and cardiovascular health. Frequency and session length are aspects linked to the design of the training program which could impact the results achieved by researches. An exercise program comprised of 2 sessions/week of between 10-15 minutes [24]. required a greater high-intensity duration for modifying measurements of either cardiorespiratory or metabolic characteristics than those comprised of 25-35-minute sessions [44], 20-35 minutes [48], 2025 minutes [45], or 30 minutes [43].

The authors can state that the exercise program including the intensity of $60-70 \%$ of HRmax should be performed to show significant improvement. Individuals with DS exhibit autonomic dysfunction, which results in reduced HRmax [20,23] Readers could think if four revised studied $[44-46,48]$ used age estimated HRmax, 60-70\% exercise intensity will induce higher exercise intensity than what it supposed to be DS. However, [65] investigated heart rate validity at repose and during exercise in people with DS and concluded that this population has greater parasympathetic activity at repose, but group differences vanish with the beginning of exercise, which advises that other variables such as reduced catecholamine sensitivity or circulating catecholamines could be answerable to chronotropic incompetence in persons with DS. 
In exercise, lipids are an important source of energy; demand increases as the duration of the exercise is prolonged [66]. Insulin resistance and obesity, which are recurrent among people with DS, are linked to harmful (more atherogenic) lipid profiles, characterized by elevated triglycerides and low HDL cholesterol [67]. This alteration in the lipid profile contributes to a higher rate of myocardial infarction and cerebrovascular accidents than in a population without DS. Previous findings paralleling lipoprotein and lipid concentrations in people with and without DS resulted in conflicting outcomes $[68,69]$. Furthermore, it is uncertain whether people with DS have an especially atherogenic lipid profile before evolving diabetes and obesity [67]. On the other hand, changes in production of thyroid hormones (hypotiroidism) can influence the lipid profile of this population [70]. Thus, to ensure that triglyceride and cholesterol levels remain at the correct levels, monitoring thyroid hormone level is important.

In their research, [46] found that the percentage of fat mass and low-density cholesterol lipoprotein (LDL) levels decreased very significantly in DS youths subjected to a strength training circuit program. In turn, an increase in HDL cholesterol levels was observed in addition to a reduction in fat mass. The latter prevents obstruction of the arteries and transports excess cholesterol to the liver so that it can be excreted, preventing heart disease [71]. In people with DS, a diminution in HDL cholesterol and an increase in body mass is linked to the development of metabolic syndrome [72]. Therefore, we can state that lipoperoxidation could be predicted in other contexts, given that in this study the control group consisted of people with SD, adjusted for age and sex, thus avoiding the bias caused by using controls without Trisomy- 21 .

Although to prevent oxidative damage a balance is necessary between enzyme activity in the first and second steps (that is, the quotient of SOD/GPX + catalase), overexpression of the SOD gene located in chromosome 21 would lead to an excess in the production of H2O2, which could transform into hydroxyl radicals. This fact would explain, at least in part, the higher sensitivity of trisomic cells to oxidative injury [73]. However, instead of a rise in SOD activity being favorable, augmented lipid peroxidation is linked to increased expression; similarly, researches on transgenic animals and bacteria indicate that high levels of SOD produce an increase in lipid peroxidation and hypersensitivity to oxidative stress [73]. Erythrocytes, which contain a high content of antioxidant enzymes, provide a great deal of information about physiological processes occurring in other tissues; this information can be accessed using a minimally invasive technique [74]. Therefore, [44], studied the effect of regular exercise on erythrocyte catalase activity in the DS population with the purpose of increasing their redox metabolism. They concluded that the 12 -week program of physical activity increased the catalase erythrocyte, although not significantly. In spite of the fact that studies of individuals with intellectual disability have not been found. [75] statement a significant increase in activity of the catalase enzyme in patients with arterial hypertension after a 3-month program has been published.

Lipid peroxidation is a deteriorating process that occurs under oxidative stress, altering cell membranes, lipoproteins and other structures containing cholesterol, glycolipids and unsaturated phospholipids [76]. In proposed a study to discover the impact of a strength training circuit program on lipoperoxidation in sedentary adults with DS. Their results showed that lipoperoxidation was reduced; after the training program, plasma ox-LDL levels decreased due to the improvement of enzyme-induced antioxidant systems caused by exercise at light/moderate intensities $[77,78]$.

Malondialdehyde is a final product of the oxidation of polyunsaturated fatty acids. It is used as an oxidative degradation marker in the cell membrane $[48,79,80]$ observed that plasma levels of malondialdehyde decrease significantly in individuals with DS who exercise regularly. This decrease indicates that there is a decline in oxidative stress, improving the antioxidant system and protecting against the damage those free radicals can cause [81]. Allantoin is a water-soluble product that is easily eliminated by the kidneys. It is composed of two urea molecules that, together with ammonia and uric acid, contribute to the excretion of excess nitrogen. [45] used a 12-week aerobic training program for adolescents with DS; they observed a reduction in plasma levels of allantoin and the allantoin/uric acid ratio after exercise. This may be due to the fact that regular exercise was performed at a low to moderate intensity that improved the activity of antioxidant enzymes, which dampens the oxidation of uric acid.

It has been observed that in individuals with DS who exercise daily, elimination of uric acid in urine is reduced; this could be due in part to the increase in free radicals and reactive oxygen species generated with physical exercise. The quality of life of this population improves as results of a decrease in uric acid [82]. Three studies $[44,45,48]$ only measure a single physiologic parameter related to oxidative stress. Thus, after assessing their results it could be said that physical activity has a useful effect on people with DS who participate in a 12-weeks exercise program [83-88]. However, we propose the effect of exercise on physiological parameters that indicate oxidative stress should be studied in greater depth [89-91].

\section{Strengths}

In terms of strengths, we may say this review offers a more complete view of different training programs than a single article can provide. This systematic review addresses an important topic and will help to inform the approach to beneficial physical activity programs for people with DS. This review also offers a major contribution to improving the wellbeing of people with DS through exercise [92]. Physiological benefits of exercise programs are an important issue as can be used to create new questions of form hypothesis about exercise physiology in people with DS. This 
investigation has "ecological validity" because the research results may be generalized to other conditions, different contexts, different researchers etc.

\section{Limitations}

The particular limitations of the present review must be emphasized. On the one hand, we paid attention to articles published in databases, not including annual activities or reports, conference proceedings, newsletters, etc. On the other hand, we focused on aerobic workouts lasting between 12 and 30 weeks, considering that less time does not imply appreciable physiological change in the participants. The key words chosen may have limited our review. In assessing the resulting data quality of this review, readers should take into account that we did not examine for articles previous to 2000. Nonetheless, we followed the bibliometric criteria suggested by Juan [49]. Though only six databases were used, they covered the subject matter very well. The search was carried out in duplicate to decrease the option of incorrectly eliminating important studies. The response of lipid profiles to exercise was only evaluated in one article; data relating to individuals with DS was taken into account, and those referring to other intellectual disabilities were excluded. Several studies were carried out by the same groups of researchers; therefore, there may be some overlap of participants in the various training programs. Our review includes a relatively small number of studies; therefore, caution must be employed in generalizing with regard to the results.

\section{Conclusion}

Physiological benefits of exercise programs are an important issue as can be used to create new questions of form hypothesis about exercise physiology in people with DS. It has been proven that different training programs have physiological benefits in young people with DS. However, as demonstrated in selected studies, to increase cardiorespiratory fitness, decrease oxidative stress levels and improve lipid profiles and antioxidant capacity, it would be advisable to use an aerobic training program for a minimum of 12 weeks duration, with three sessions/week at $60-75 \%$ HRmax, 30 -minutes section. In case of need, short rests must be included; these will be reduced as the individual begins to accept continued exercise. At least three sessions/week is recommended, because physical of condition people with DS is often deficient as well, making it extremely difficult to plan several low-intensity workouts.

Taking int account our findings, researchers should focus on an in-depth study of lipid profiles in this population, because although beneficial results have been observed, it is true that the effects on lipid metabolism are reversible and may disappear. Therefore, people with DS should incorporate physical exercise into their lifestyle. We encourage researcher to perform high-quality studies by means of assessing more than one physiological parameter in plasma and blood samples and including the thyroid hormones evaluation. From the point of view of obsolescence of the studies, it is very interesting and necessary that researchers continue to delve into the physiological benefits of exercise programs in people with DS.

\section{Authors Contribution}

Conceptualization, R.C., F.R., E.I.A. and C.C.R.; Methodology, R.C., F.R., E.I.A. C.C.R. Validation, R.C., C.C.R., E.I.A., J.C., F.R., B.E., A.M and M.D.R. Formal Analysis, R.C, F.R. and J.C.; Investigation, R.C., J.C and F.R.; Resources, R.C. and F.R.; Data Curation, R.C. and F.R. Writing - Original Draft Preparation, R.C.. F.R. Original Draft Revision: R.C., E.I.A., F.R. Writing - Review \& Editing, R.C., E.I.A. F.R.; Supervision, E.I.A and C.C.R. All authors have read and agree to the published version of the manuscript.

\section{Funding}

This research lacks external funding.

\section{Disclosure Statement}

No potential conflict of interest is reported.

\section{Acknowledgment}

Authors are grateful to Dr. Maria Dolores Rubio for scientific advice and suggesting the views for the assessment of methodological quality and risk of bias of the studies included in the review.

\section{References}

1. Gardiner KJ (2010) Molecular basis of pharmacotherapies for cognition in Down syndrome. Trends in Pharmacological Science 31: 66-73.

2. Robles MA (2007) Incidencia y prevalencia del Síndrome de Down. Revista Síndrome de Down: Revista Española de Investigación e Información sobre el Síndrome de Down 93: 68-70.

3. Antonarakis SE, Skotko BG, Rafi MS, Strydom A, Pape SE, et al. (2020) Down syndrome. Nature Reviews Disease Primers 6(1): 9.

4. Coskun PE, Busciglio J (2012) Oxidative stress and mitochondrial dysfunction in Down syndrome: relevance to aging and dementia. Current Gerontology and Geriatrics Research 57: 1-7.

5. Penedo FJ, Dahn JR (2005) Exercise and well-being: a review of mental and physical health benefits associated with physical activity. Journal of Behavioral Medicine Med 18: 189-193.

6. Rowell LB, O’Leary DS, Kellogs DL (1996) Handbook of physiology exercise: regulation and integration of multiple system. USA: Bethesa M.D.

7. Carnethon MR, Gidding SS, Nehgme R, Sidney S, Jacobs DR, et al. (2003) Cardiorespiratory fitness in young adulthood and the development of cardiovascular disease risk factors. Journal of American Medical Association 290: 3092-3100.

8. Wen CP, Wai JP, Tsai MK, Yang YC, Cheng TY, et al. (2011) Minimum amount of physical activity for reduced mortality and extended life expectancy: a prospective cohort study. Lance 378: 1244-1253.

9. Agiovlasitis S, Choi P, Allred AT, Xu J, Molr RW (2019) Systematic review of sedentary behavior in people with Down Syndrome across the lifespan: A clarion call. Journal of Applied Research in Intellectual Disabilities 33(4). 
10. Alesi M (2017) Investigating parental beliefs concerning facilitators and barriers to the physical activity in Down syndrome and typical development. SAGE Open 7(1).

11. Glasson EJ, Sullivan SG, Hussain R, Petterson BA, Montgomery PD, et al. (2002) The changing survival profile of people with Down's syndrome: implications for genetic counseling. Clinical Genetics 62: 390-393.

12. Tenenbaum A, Chavkin M, Wexler ID, Korem M, Merrick J (2012) Morbidity and hospitalizations of adults with Down syndrome. Research in Developmental Disabilities 33: 435-441.

13. Standcliffe RJ, Lakin KC, Larson S, Engler J, Bershadsky J, et al. (2001) Overweight and obesity among adults with intellectual disabilities who use intellectual disability/developmental disability service in 20 U.S. states. American Journal on Intellectual and Developmental Disabilities 116: 401-418.

14. Hermon C, Alberman E, Beral V, Swerdlow AJ (2001) Mortality and cancer incidence in persons with Down's syndrome, their parents and siblings. Annals of Human Genetics 65: 167-176.

15. Barr M, Shields N (2011) Identifying the barriers and facilitators to participation in physical activity for children with Down syndrome. Journal of Intellectual Disabilities Research 55: 1020-1033.

16. Draheim CC, Williams DP, McCubbin JA (2002) Prevalence of physical inactivity and recommended physical activity in community-based adults with mental retardation. Mental Retardation 40: 436-444.

17. Waden TA, Webb VL, Moran CH, Bailer BA (2012) Life modification for obesity. New developments in diet, physical activity, and behavior therapy. Circulation 125: 1115-1170.

18. Shields N, Taylor NF (2015) The feasibility of physical activity program for young adults with Down syndrome: A phase II randomized controlled trial. Journal of Intellectual Developmental Disability 40: 115-125.

19. Mc Ardle W, Katch V, Katch F (2015) Fisiología del ejercicio, nutrición y rendimiento. (8 ${ }^{\text {th }}$ Edn.,)., Madrid. Wolters Kluwer.

20. Guerra M (2000) Síndrome de Down y respuesta al esfuerzo físico Doctoral thesis. Escola de Medicina de l’Educació Física i l’Esprot. Barcelona.

21. Hardee JO, Fetters L (2017) The effect of exercise intervention on daily life activities and social participation in individuals with Down syndrome: a systematic review. Research in Developmental Disabilities 62: 81-103.

22. Díaz-Cuéllar S, Yokoyama-Rebollar E, Del Castillo-Ruíz V (2016) Genomics of Down Syndrome. Acta Pediatrica de México 37: 289-296.

23. Fernhall B, Pitetti KH, Rimmer JH, McCubbin JA, Rintala P, et al. (1996) capacity of individuals with mental retardation including Down syndrome. Medicine \& Science in Sports Exercise 28: 366-371.

24. González-Agüero A, Gómez-Cabello A, Matute-Llorente A, GómezBruton A, Vicente-Rodríguez G, et al. (2014) Efectos del entrenamiento pliométrico sobre la resistencia cardiorrespiratoria de niños y adolescentes con síndrome de DownEffects of a circuit training including plyometric jumps on cardiorespiratory fitness of children and adolescents with Down syndrome. Revista Medica Internacional sobre Síndrome de Down 18: 35-42.

25. Casajus JA, Pueyo D, Vicente-Rodríguez G, González-Agüero A (2012) Mejoras de la condición cardiorrespiratoria en jóvenes con síndrome de Down mediante entrenamiento aeróbico: estudio longitudinalImprovements in cardiorespiratory fitness through aerobic training in young people with Down's syndrome: a longitudinal study. Medicina de l'Esport 47(154): 49-54.

26. Ortega FB, Ruiz JR, Castillo MJ, Sjöström M (2008) Physical fitness in childhood and adolescence: a powerful marker of health. International Journal of Obesity 32: 1-11.
27. Poole DC, Wilkerson DP, Jones AM (2008) Validity of criteria for establishing maximal 02 uptake during ramp exercise tests. European Journal of Applied Physiology 102: 403-410.

28. Cornish AK, Broadbent S, Cheema BS (2011) Interval training for patients with coronary artery disease: A systematic review. European Journal of Applied Physiology 11: 579-589.

29. Chomistek AK, Cook NR, Rimm EB, Ridker PM, Buring JE, et al. (2017) Physical activity and incident cardiovascular disease in women: is the relation modified by level of global cardiovascular risk? Journal of American Heart Association 7(12): e008234.

30. González de Buitrago JM, Arilla Ferreiro E, Rodríguez-Sedade M, Sánchez Pozo A (1998) Bioquímica Clínica. Madrid. Editorial McGraw-HillInteramericana.

31. Sobey CG, Judkins CP, Sundararajan V, Phan TG, Drummond GR, et al. (2015) Risk of major cardiovascular events in people with Down syndrome. PLoS ONE 10: e0137093.

32. Hill DA, Gridley G, Cnattingius S, Mellemkjaer L, Linet M, et al. (2003) Mortality and cancer incidence among individuals with Down syndrome. Archives of International Medicine 163: 705-711.

33. De la Piedra MJ, Alberti G, Cerda J, Cárdenas A, Paul MA, et al. (2017) High frequency of dyslipemia in children and adolescents with Down Syndrome. Revista Chilena de Pediatría 88(5): 595-601.

34. Mann S, Beedie C, Jiménez A (2014)Differential Effects of Aerobic exercise, resistance training and combined exercise modalities on cholesterol and the lipid profile: Review, Synthesis and Recommendations. Sports Medicine 44: 211-221.

35. Slater TF (1984) Free radical mechanism in tissue injury. Biochemical Journal 222: 1-15.

36. Muchová J, Zitnanová I, Duracková Z (2014) Oxidative stress and Down syndrome. Do antioxidants play a role in therapy? Physiology Research 63: 535-542.

37. De Haana JB, Susil BJ, Pritchard M, Kola I (2003) An altered antioxidant balance occurs in Down syndrome fetal organs: implications for the gene dosage effect hypothesis. Journal Neural Transmission Supplementa 67: 67-83.

38. Fato R, Bergamini C, Leoni S, Lenaz G (2008) Mitochondrial production of reactive oxygen species: role of complex I and quinone analogues. Biofactors 32: 31-39.

39. Koopan WJ, Nitjmans LG, Dierten CJ, Roestenberg P, Valscchi F, et al. (2010) Mammalian mitochondrial complex I: biogenesis, regulation and reactive oxygen species generation. Antioxidants \& Redox Signaling 12: 1431-1470.

40. Juan V (2018) Publicar con impacto. Cómo analizamos la producción científica. Biblioteca Virtual del Sistema Sanitario Público de Andalucía.

41. Moseley AM, Herbert RD, Sherrington C, Maher CG (2002) Evidence for physiotherapy practice: A survey of the Physiotherapy Evidence Database (PEDro). Australian Journal of Physiotherapy 48: 43-49.

42. Varela AM, Sardinha LB, Pitetti KH (2001) Effects of an aerobic rowing training regimen in young adults with Down syndrome. American Journal of Mental Retardation 106: 135-144.

43. Tsimaras V, Giagazoglou P, Fotiadou E, Christoulas K, Angelopoulou N (2003) Jog-walk training cardio-respiratory fitness of adults with Down syndrome. Perceptual and Motor Skills 96(3): 1239-1251.

44. Rodríguez M, Rosety M, Ordoñez FJ (2006) Influence of regular exercise on erythrocyte catalase activity in adolescents with Down syndrome. Medicina Clínica 127: 533-534.

45. Rosety-Rodríguez M, Rosety I, Fornieles-González G, Díaz A, Rosety M, et al. (2010) A 12-week aerobic training programme reduced plasmatic 
allantoin in adolescents with Down syndrome. British Journal of Sports Medicine 44: 685-687.

46. Rosety-Rodríguez M, Fornieles G, Rosety MA, Díaz AJ, Rosety I, etal. (2013) Mejora del perfi 1 lipídico y lipoperoxidación mediante entrenamiento de fuerza en adultos con discapacidad intelectual. Archivos de Medicina del Deporte 30: 256-259.

47. Miralles J, Ramos JM, Ballester R, Belinchón I, Sevila A, et al. (2005) Estudio bibliométrico de la revista Actas Dermo-Sifiliográficas (19842003) II. Análisis de las referencias bibliográficasBibliometric study of the journal actas dermo-sifiliográficas (1984-2003) II. analysis of bibliographical references. Actas Dermo-Sifiliográficas 96: 563-571.

48. Ordóñez FJ, Rosety-Rodríguez M (2007) Regular exercise attenuated lipid peroxidation in adolescents with Down's syndrome. Clinical Biochemistry 40: 141-142.

49. Gorbea-Portal S (2007) Comportamiento de la obsolescencia en dos revistas mexicanas. In: Martínez FF, Calva JJ (Eds.).), 25 años de investigación en ciencias bibliotecológicas y de la información. Ciudad de México: Universidad Nacional Autónoma de México.

50. Sotelo-Cruz N, Atrián-Saazar ML, Trujillo-López S (2016) Indicators of obsolescence of the medical literature in a Mexican pediatric journal. Gaceta Médica Méxica 152: 181-185.

51. Topcu H, Arabaci R (2017) Acute effect of different warm up protocols on athlete's performance. European Journal of Physical Education and Sport Science 3: 35-50.

52. López Chicharro (2019) Fisiología del Ejercicio. (3 ${ }^{\text {rd }}$ Edn.), Madrid. Panamericana.

53. Ewing G, McDermott S, Thomas-Koger M, Whitner W, Pierce K (2004) Evaluation of a cardiovascular health program for participants with mental retardation and normal learners. Health Education and Behavior: The Official Publication of the Society for Public Health Education 31: 77-87.

54. Allender S, Cowburn G, Foster C (20006) Understanding participation in sport and physical activity among children and adults: a review of qualitative studies. Health Education Research 21: 826-835.

55. Rees R, Kavanagh J, Harden J, Shepherd J, Brunton G, et al. (2006) Young people and physical activity: a systematic review matching their views to effective interventions. Health Education Research 21: 806-825.

56. May J, Shields N, Taylor F, Dodd KJ (2011) Factores que dificultan la actividad física de los adultos con síndrome de Down. Revista Síndrome de Down 108: 34-45.

57. Baynard T, Pitetti KH, Guerra M, Unnithan VB, Fernhall B (2008) Age-related changes in aerobic capacity in individuals with mental retardation: A 20-yr review. Medicine \& Science in Sports \& Exercise 40 1984-1989.

58. Guerra M, Gine-Garriga M, Fernhall B (2009) Reliability of Wintage testing in adolescents with Down syndrome. Pediatric Exercise Science 21: 47-54.

59. Mercer VS, Lewis CL (2001) Hip abductor and knee extensor muscle strength of children with and without Down syndrome. Pediatric Physical Therapy 13: 18-26.

60. González-Agüero A, Ara I, Moreno LA, Vicente-Rodríguez G, Casajus T (2011a) Fat and lean masses in youths with Down syndrome: Gender differences. Research in Developmental Disabilities 32: 1685-1693.

61. Conley RW (1985) Down syndrome: economic burdens and benefits of prevention. Basic Life Science 36: 35-59.

62. González-Agüero A, Vicente- Rodríguez G, Moreno LA, Casajus JA (2011b) Bone mass in female and male adolescents with Down Syndrome. A Peripheral Quantitative Computed Tomography (pQCT) study. Osteoporosis International 22: 2151-2157.
63. Morgan DW, Bransford DR, Costill DL, Daniels JT, Howley ET, et al. (1995) Variation in the aerobic demand of running among trained and untrained subjects. Medicine and Science in Sports \& Exercise 27: 404-409.

64. Vicente-Rodríguez G, Ara I, Pérez-Gómez J, Dorado C, Calbet JA (2005) Muscular development and physical activity as major determinants of femoral bone mass acquisition during growth. British Journal of Sports Med 39: 611-616.

65. Baynard T, Pitetti KH, Guerra M, Fernhall B (2004) Hear rate variability at rest and during exercise in person with Down syndrome. Comparative study 85: 1285-1290.

66. Fernández A (2006) Respuesta cardíaca al ejercicio. In: López Chicharro; Fernández Vázquez (Eds.).), Fisiología del Ejercicio. 3ạ España. Panamericana pp. 321-330.

67. Adelekan T, Magge S, Shults J, Stallings V, Stettler N (2012) Lipid profiles of children with Down syndrome compared with their siblings. Pediatrics 129: e1382-e1387.

68. Murdoch JC, Rodger JC, Rao SS, Fletcher CD, Dunnigan MG (1977) Down's syndrome: an atheroma-free model? British Medical Journal 2: 226-228.

69. Nishida Y, Akaoka I, Nishizawa T, Maruki M, Maruki K (1977) Hyperlipidaemia in patients with Down's syndrome. Atherosclerosis 26 : 369-372.

70. Werle de Almeida E, Gregoul M (2020) Lipid profile in people with Down syndrome: a literature review. Journal of Human Growth and Development 30: 197-208.

71. Laris MR, Arteaga A, Cueva A, Attilio R (2005) El colesterol HDL: ¿un nuevo objetivo terapéutico en el manejo de las dislipidemias y la ateroesclerosis? Revista Médica de Chile 133: 823-832.

72. Soler A, Xandri JM (2011) Nutritional status of intellectual disabled persons with Down syndrome. Nutrición Hospitalaria 26: 1059-1066.

73. Kowald A, Klipp E (2004) Alternative pathways might mediate toxicity of high concentrations of superoxide dismutase. Annals of the New York Academy of Science 1019: 370-374.

74. Muchová J, Sustrová M, Garaiová I, Liptáková A, Blazícek P, et al. (2001) Influence of age on activities of antioxidant enzymes and lipid peroxidation products in erythrocytes and neutrophils of Down syndrome patients. Free Radical Biology and Medicine 31: 499-508.

75. Chirac C, Ungureanu D, Dima-Cozma C, Jerca O, Iacobovici A, et al. (2004) Evaluation of oxidative stress and enzymatic antioxidants in medium physical training of moderate arterial hypertension. Revista medicochirurgicala a Societatii de Medici si Naturalisti din Iasi 108: 74-78.

76. Tsimikas S (2008) In vivo markers of oxidative stress and therapeutic interventions. American Journal of Cardiology 101: 34D-42D.

77. Monteiro CP, Varela A, Pinto M, Neves J, Felisberto GM, et al. (1997) Effect of an aerobic training on magnesium, trace elements and antioxidant systems in a Down syndrome population. Magnesium Research 10: 6571.

78. Bosy-Westphal A, Schautz B, Later W, Kehayias JJ, Gallagher D, et al. (2013) What makes a BIA equation unique? Validity of eight-electrode multifrequency BIA to estimate body composition in a healthy adult population. Europena Journal of Clinical Nutrition 67: 14-21.

79. Gonenc S, Acikgoz O, Semin I, Ozgonul H (2000) The effect of moderate swimming exercise on antioxidant enzymes and lipid peroxidation levels in children. Indian Journal of Physiology and Pharmacology 44: 340-344.

80. Muñiz P, Coma MJ, Terán J (2014) Estrés oxidativo y daño vascular de hipoxia. Malondialdehido (MDA) como biomarcador de daño oxidativo. Electronic Journal of Biomedicine 2: 46-49.

81. Casado A, López-Fernández E, Castellanos A (2014) El ejercicio físico disminuye el estrés laboral y oxidativo en profesionales de urgencias. Revista de Laboratorio Clinico 7(3): 96-103. 
82. Campos C, Guzmán R, López-Fernández E, Casado A (2013) Physical exercise and urinary uric acid levels in Down's syndrome. Revista Médica Internacional de Síndrome de Down 17: 1-5.

83. Banky M, Shields N (2011) Identifying the barriers and facilitators to participation in physical activity for children. Journal of Intellectual Disabilities Research 55: 1020-1033.

84. González-Agüero A, Vicente-Rodríguez G, Moreno LA, Guerra M, Ara I, et al. (2010) Health-related physical fitness in children and adolescents with Down syndrome and response to training. Scandinavian Journal of Medicine and Science in Sports 20: 716-724.

85. Guerra M, Llorens N, Fernhall B (2003) Chronotropic incompetence in individuals with Down syndrome. Archives of Physical Medicine and Rehabilitation 84(11): 1604-1608.

86. Kazemi M, Salehi M, Kheirollahi M (2016) Down syndrome: current status and future perspectives. International Journal of Molecular and Cellular Medicine 5: 125-133.

ISSN: 2574-1241

DOI: 10.26717/BJSTR.2020.31.005105

Agüera EI. Biomed J Sci \& Tech Res

(C) This work is licensed under Creative

Submission Link: https://biomedres.us/submit-manuscript.php
87. La Course L (2009) Physical Activity in Individuals with Down Syndrome: An Overview, and Suggestions for Appropriate Activities and Instructional Techniques. Syracuse University Honors Program Capstone Projects p. 456

88. Maher CG, Sherrington C, Herbert RD, Moseley AM, Elkins M (2003) Reliability of the PEDro Scale for Rating Quality of Randomized Controlled Trials. Physical Therapy 83: 713-721.

89. PEDro.

90. Wee SO, Pitetti K, Goulopoulou S, Collier S, Guerra M, et al. (2015). Impact of obesity and Down syndrome on peak heart rate and aerobic capacity in youth and adults. Research in Developmental Disabilities 36: 198-206.

91. WHO.

92. (2004) World Health Organization. Global strategy on diet, physical activity and health. Geneva: World Health Organization.

$\begin{array}{ll}\text { BIOMEDICAL } & \text { Assets of Publishing with us } \\ \text { RESEARCHES } & \text { - Global archiving of articles } \\ \text { - Immediate, unrestricted online access }\end{array}$

